\title{
Exploring relationship between crude oil price volatility and stock indices movement using wavelet analysis: evidence from India and China
}

\author{
Shekhar Mishra \\ Department of Business Management, C.V. Raman Global University, \\ Bhubaneswar, India, and \\ Sathya Swaroop Debasish \\ Department of Business Administration, Utkal University, Bhubaneswar, India
}

Crude oil price volatility

Received 17 November 2020 Revised 19 December 2020 Accepted 19 December 2020

\begin{abstract}
Purpose - This study aims to explore the linkage between fluctuations in the global crude oil price and equity market in fast emerging economies of India and China.

Design/methodology/approach - The present research uses wavelet decomposition and maximal overlap discrete wavelet transform (MODWT), which decompose the time series into various frequencies of short, medium and long-term nature. The paper further uses continuous and cross wavelet transform to analyze the variance among the variables and wavelet coherence analysis and wavelet-based Granger causality analysis to examine the direction of causality between the variables.

Findings - The continuous wavelet transform indicates strong variance in WTIR (return series of West Texas Instrument crude oil price) in short, medium and long run at various time periods. The variance in CNX Nifty is observed in the short and medium run at various time periods. The Chinese stock index, i.e. SCIR, experiences very little variance in short run and significant variance in the long and medium run. The causality between the changes in crude oil price and CNX Nifty is insignificant and there exists a bidirectional causality between global crude oil price fluctuations and the Chinese equity market.

Originality/value - To the best of the authors' knowledge, very limited work has been done where the researchers have analyzed the linkage between the equity market and crude oil price fluctuations under the framework of discrete wavelet transform, which overlooks the bottleneck of non-stationarity nature of the time series. To bridge this gap, the present research uses wavelet decomposition and MODWT, which decompose the time series into various frequencies of short, medium and long-term nature.
\end{abstract}

Keywords MODWT, Crude oil price, CNX Nifty, Continuous wavelet transform, Shanghai Composite Index, Wavelet-based Granger causality analysis

Paper type Research paper

(C) Shekhar Mishra and Sathya Swaroop Debasish. Published in Vilakshan - XIMB Journal of Management. Published by Emerald Publishing Limited. This article is published under the Creative Commons Attribution (CC BY 4.0) licence. Anyone may reproduce, distribute, translate and create derivative works of this article (for both commercial and non-commercial purposes), subject to full attribution to the original publication and authors. The full terms of this licence maybe seen at http:// creativecommons.org/licences/by/4.0/legalcode

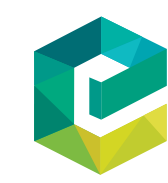

Vilakshan - XIMB Journal of Management Vol. 19 No. 1, 2022 pp. $69-86$

pp. $69-86$
Emerald Publishing Limited 0973-1954 DOI 10.1108/XJM-11-2020-0204 
$\mathrm{XJM}$

19,1

\section{Introduction}

The linkage between the fluctuations in crude oil price and equity market has received significant thoughtfulness among industry practitioners and academicians globally. Oil plays a crucial role in shaping the economic development of the countries especially the emerging ones across the globe. The fact gains more prominence as more than half of world's total oil consumption is made by emerging economies (Pershin et al., 2016). During recent times, the study of influence of oil price fluctuations on the equity market has gained significance as the analysis of oil-stock market linkage plays a crucial role in asset allocation and portfolio risk management decisions. This may be owed to the fact that the movement in equity is not only dependent upon prevailing fundamental information but also contingent upon the oil market information (Mensi et al., 2017). The shocks in oil price influence the stock market price by influencing the expected earnings (Jones and Kaul, 1996). The positive or negative linkage between the variables depends upon the nation's prevailing economic conditions. The same is also contingent upon whether the crude oil price is driven by demand shocks or supply shocks. Further, the rise in oil price has debilitating effect on oil importing nations and the same is favorable for oil exporting economies.

Fayoumi (2009) found the negative linkage between the variables because of detrimental effect of oil price increase on the company's earnings, which further cause decline in their profit and dividends considered to be key driver of the equity prices. The negative effects of oil price volatility on the stock market of oil importing nations could be observed as they bear the increased cost and face rise in risk and uncertainty. The inflationary pressure of oil price rise also calls for intervention by regulators by raising interest rates to control the inflation. According to equity pricing model (Basher et al., 2012), the increased interest rates negatively influence the discount rate and thus affects the present value of future cash flows. The decreased present value of imminent cash flows further stimulates the stock price decline (Jones et al., 2004). The rise in interest rates drive the investors away from equity market toward bond investments thus leading to further fall in stock prices (Basher and Sadorsky, 2006).

The recovery from economic recession stimulates rise in global demand thus leading to increase in basic production input costs such as that of oil price. In this scenario, the rise in oil price may have a favorable effect on equity returns. In oil exporting countries, the oil price rise positively influences equity market through wealth and income effects. In oil exporting countries, the increased oil price may lead to enhanced revenue for their respective government, and increased public spending on infrastructure. The wealth transfer from oil importers to oil exporters because of oil price rise may enhance the economic activity and thus improve the returns in stock index.

\section{Literature review}

The findings of the literature analyzing the influence of global crude oil price fluctuations on equity market have not been able to arrive at the consensus. The linkage between the variables has been either observed to positive or negative. Jones and Kaul (1996) found the negative impact of oil price shocks on aggregate stock returns of USA, Canada, UK and Japan. O'Neil et al. (2008) also documented negative relationship between the variables for the US, UK and France equity market. The outcome was supported by findings of Sadorsky (1999) for the monthly data for US equity market. Similarly, Valadkhani et al. (2009) while using GARCH-M model found negative linkage between oil price fluctuations and Thai stock market in pre-Asian crisis period. Pappetrou (2001) and Filis (2010) also observed the same for economic conditions of Greece. 
Driesprong et al. (2008) found oil price changes having significant predictability on stock market returns for the majority of developed and emerging countries examined. For understanding the dynamics between the given variables in emerging markets, Basher et al. (2012) while applying structural vector autoregression (VAR) model found positive oil price shocks negatively influencing the equity returns. Cunando and De Gracia (2014) with VAR and vector error-correction model methodology observed remarkable negative effect of oil price changes on stock market returns in most oil importing European countries taken under study. In the industry level analysis, Nandha and Faff (2008) examined the impact of rise in oil price on 35 global industry indices and observed equity returns of industries, except that of mining and oil and gas industries, being negatively influenced by oil price increase.

The number of researchers has also supported the positive association between oil price shocks and stock returns in their study. Sadorsky (2001) applied multifactor model to found positive linkage between oil price increase and stock returns of Canadian oil and gas industries. The findings were also supported by the study of Boyer and Filion (2004) for Canadian energy stocks and overall markets returns. Hammoudeh and Li (2005) found oil price growth positively linked with equity returns of oil sensitive industries of USA and stock returns of oil exporting countries. For 21 emerging markets taken under study, Basher and Sadorsky (2006) found oil price risk to be positively linked with the pricing of emerging market stock returns. Gogineni (2007) and Yurtsever and Zahor (2007) observed positive association between stock prices and oil price shocks which reflected changes in aggregate demand. However, the relationship was observed to be negative where oil price shock reflected changes in aggregate supply. Bjorland (2008) analyzed the indirect effect of oil price changes on stock prices through monetary policy responses and demonstrated positive effect of oil price increases on stock returns in Norway. The author concluded that higher oil prices increase stock returns through economy's response to price increase by increasing aggregate wealth and demand. Narayan and Narayan (2010) used co integration method and observed positive long-run linkage between Vietnamese equity returns and oil price fluctuations. In Gulf Cooperation Council countries except Kuwait, Mohanty et al. (2011) observed positive and significant relationship between oil price changes and stock prices.

In the industry level analysis for Australia, Faff and Brailsford (1999) found positive oil price sensitivity of oil, gas and diversified resources industries. In the similar fashion, El-Sharif et al. (2005) found oil price fluctuations positively influencing UK energy companies' equity prices.

The study of Lee and Chang (2011) revealed the varied yet significant reaction of stock markets to oil price shocks across the Asian markets. The authors observed positive response of stock market to oil price in Japan, while the same was negative in Malaysia. For Singapore and South Korea, the outcome was ambiguous. Similarly, Lin et al. (2010) also found positive effect of oil price shocks for Hong Kong and negative for Taiwan.

Further, in China, Cong et al. (2008) found significant effect of oil price shocks on mining, petrochemicals and manufacturing index, and select oil companies' equity returns. The bidirectional causality between change in crude oil price and stock market returns was found by Anoruo (2010). On the contrary, in the earlier study of Anoruo and Mustafa (2007), long-term relationship between the variables and one way causality relationship from stock market returns to oil returns was observed. In South Korea, Masih et al. (2011), while applying VAR model to examine the inter relationship between the given variables, observed real stock returns being significantly influenced by oil price volatility. In Indian economic scenario, Chittedi (2012) and Sahu et al. (2014) found co integrating linkage and long-term co-movement between the changes in oil prices and stock price fluctuations. 
$\mathrm{XJM}$

19,1

Similarly, Toraman et al. (2011) and Sharma and Khanna (2012) observed long-term relationship between stock market indices and oil price for the Asia-Pacific countries.

Bhar and Nikolova (2009) found time varying correlation between oil price fluctuations and stock market volatility in Brazil, Russia, India, China and South Africa and the outcome of their study is contingent upon whether the particular country is net oil exporter or oil importer. The authors' study was based upon the works of Gay (2008) and Benassy-Quere et al. (2007). A strong unidirectional causality running from oil prices to stock prices was observed by Lescaroux and Mignon (2008) in their study for oil exporting countries.

Many authors also failed to find any significant linkage between changes in crude oil price and stock price volatility in their analysis. Chen et al. (1986), Huang et al. (1996) and Wei (2003) did not find any significant linkage between the variables in their analysis. Maghyereh (2004), while analyzing the interaction between oil price shocks and stock markets in a broad sample of 22 emerging markets, found that these oil price shocks do not have significant influence on stock index returns. Similarly, Sari and Soytas (2006), Cong et al. (2008) and Sehgal and Kapur (2012) also did not observe oil price shocks having any significant impact on real stock returns.

The majority of the literature examining the inter-linkage between oil prices and stock market behavior has remained confined to the conventional techniques such as general auto regressive conditional heteroskedasticity class models, VAR, cointegration and the Granger causality methods. The outcome from these models applied by the researchers failed to arrive at the consensus. Further, these techniques require time series to be of stationary in nature, whereas neither the oil price nor the exchange rate nor the stock indices are stationary in nature. To the best of our knowledge, very limited work has been done, where the researchers have analyzed the same under the framework of discrete wavelet transform (DWT), which overlooks the bottleneck of non-stationarity of the time series. To bridge this gap, the present research uses wavelet decomposition and maximal overlap discrete wavelet transform (MODWT), which decompose the time series into various frequencies of short, medium and long-term nature. In the preceding chapters, we discussed about theoretical background and review of literature depicting the important linkage between crude oil price, exchange rate and stock indices in the economic environment of India and China.

\section{Methodology}

The current research endeavors to explore and determine the relationship between global crude oil price and stock indices of fast emerging Asian economies of India and China for time period February 1996 to August 2019. In this regard, monthly data for stock indices comprising of China's Shanghai Composite Index (SCI) and India's National Stock Exchange (NSE) Index were sourced from official website of NSE and website of Investing.com. The Cushing OK West Texas Instrument (WTI) Spot Price, as one of the major indicators of international crude oil price, was obtained from official website of Energy Information Agency. The global crude oil price return or change can be calculated as follows:

$$
O i l_{t}=\ln \left[\frac{O i l_{t}}{O i l_{t-1}}\right]
$$

The returns from exchange rate and stock indices are also defined in a similar manner.

The paper further uses unit root tests to examine the non-stationarity of time series data which may lead to spurious regression problem and needs to be avoided. The time series data with time-varying mean or time-varying variance or both is termed to be nonstationary and in case of stationary time series data, the mean variance and auto variance remains constant (Gujarati, 2009). We use augmented Dickey-Fuller (ADF) test and Phillips-Perron (PP) test to ascertain whether the given time series data is stationary or not. 
The time and frequency domain of any time series gets combined in the wavelet analysis. Unlike other econometric methods, the wavelets decompose the time series under study into various wavelet scales. According to Ramsey (1999), the wavelets generate the orthogonal timescale decomposition of the data and give a nonparametric representation to all the individual time series (Ramsey, 1999). They have the ability to preserve the time data while performing the frequency decomposition of the series. The wavelet transform captures all the information in the time series associated with specific time horizons and time locations (Gencay et al., 2002). This feature of the wavelets makes it possible to deal with nonstationary property of the time series.

The father $(\phi)$ and mother $(\psi)$ wavelets can represent any function of time (Ramsey, 2002). The father wavelets integrate to one and represent very long scale smooth components of the signal. On the contrary, mother wavelets integrate to zero and are used to represent deviations occurring in the smooth components. Father wavelets give rise to scaling coefficients, whereas in mother wavelets, differencing coefficients are generated.

The father wavelet is represented as follows:

$$
\phi_{j, k}=-2^{-j / 2} \phi\left(\frac{t-2^{j} k}{2^{j}}\right) \text { with } \int \phi(t) d t=1
$$

The mother wavelet is represented as follows:

$$
\psi_{j, k}=-2^{-j / 2} \psi\left(\frac{t-2^{j} k}{2^{j}}\right) \text { with } \int \psi(t) d t=0
$$

The father and mother wavelets constitute the basic functions which define the sequence of coefficients. The smooth coefficients derived from the father wavelets are shown as follows:

$$
S_{j, k}=\int f(t) \phi_{J, k}
$$

The detail coefficients derived from the mother wavelet are defined as follows:

$$
d_{j, k}=\int f(t) \psi_{J, k} \text { With } j=1 \ldots \ldots \ldots \mathrm{J}
$$

The maximal scale of the former is $2^{j}$, whereas the detailed are derived from the mother wavelets at all scales from 1 to $J$. The function $f($.) from the above-mentioned coefficients can be defined as follows:

$$
f(t)=\sum_{k} S_{J, k} \phi_{J, k}(t)+\sum_{k} d_{J, k} \psi_{J, k}(\mathrm{t}) \ldots+\sum_{k} d_{J, k} \psi_{j, k}(t) \ldots+\sum_{k} d_{1, k} \psi_{1, k}(t)
$$

On simplifying equation (1), we get:

$$
f(t)=S_{J}+D_{J}+D_{J-1}+\ldots+D_{j}+\ldots+D_{1}
$$

The orthogonal components are represented as follows:

$$
S_{J}=\sum_{k} S_{J, k} \phi_{J, k}(t)
$$




$$
D_{J}=\sum_{k} d_{J, k} \psi_{J, k}(t) . \quad \mathrm{j}=1, \ldots \mathrm{J}
$$

The multi-horizon or multi-resolution breakdown of $f(t)$ is represented as $\left\{S_{J}, D_{J-1, \ldots,} D_{1}\right\} . D_{j}$ calculates the $j$ th level wavelet detail, which is related with variations in the series at scale $\lambda_{j} . S_{j}$ is defined as the cumulative sum of alterations at each level. As $j$ increases, $S_{j}$ becomes smoother and smoother (Gencay et al., 2002).

We use MODWT for estimating the scaling and wavelet coefficients. There is one of the appealing benefit of MODWT and that is, it does not suffer from any limitation such as that level of sample size to an integer multiple of $2^{J^{\circ}}$. While its limitation of DWT (Percival and Walden, 2000) is the reason that we prefer MODWT over. The detail and smooth coefficients of a MODWT are related with zero phase filters, which help in aligning the features of original time series features of multi-resolution analysis (MRA). It is considering that DWT-based estimators are asymptomatically less efficient compare to MODWT (Percival, 1995; Percival and Mofjeld, 1997; Gencay et al, 2002). Furthermore, DWT uses weighted differences and makes average of attached sets of observations, whereas MODWT uses moving difference and average operator, thus keeping the exact number of observations at each wavelet decomposition scale.

The present paper uses Daubechies least asymmetric (LA) filter of length 8 (LA8). According to Gencay et al. (2002), the LA(8) wavelet is considered to be smoother than HAAR wavelet filters which are widely used in the previous studies. According to Cornish et al. (2006), the LA(8) filter provides better uncorrelatedness across scales as compared to it HAAR filter counterpart.

We decompose the series into wavelet coefficients $D_{1}-D_{6}$. The detail coefficient $D_{j}$ gives the resolution of data at scale $2^{j}-2^{j+1}$. The oscillations of periods $2-4,4-8,8-16,16-32$, 32-64 and 64-128 months are represented by $\lambda_{1}, \lambda_{2}, \lambda_{3}, \lambda_{4}, \lambda_{5}$ and $\lambda_{6}$, respectively. The long-term movements are represented by wavelet smooth $\mathrm{S}_{6}$.

\section{Empirical analysis and discussion}

Descriptive statistics and stationarity tests. In the present research, we analyze the causality between WTI crude oil price and stock indices of India and China in time-frequency domain or more precisely wavelet framework. Prior to empirical analysis, we perform the descriptive statistics and unit root tests to analyze the univariate characteristics and stationarity of variables under study. The standard unit root tests used for ascertaining the stationarity of data comprise $\mathrm{ADF}$ and PP tests. The results of descriptive statistics and unit root tests comprising $\mathrm{ADF}$ and $\mathrm{PP}$ tests for variables at level and first-order difference are presented in Table 1.

The significant erratic behavior of the data becomes well evident from the descriptive statistics mentioned in Table 1 . The variables are observed to be left skewed indicating median to be larger than mean i.e. left-tailed distribution to be longer than right-tailed distribution. Despite positive mean indicating positive results on average yield for investors, negative skewness indicates toward more negative data being concentrated around the mean value. The negative skewness implies that the distribution bear extreme values which is further validated by large excess kurtosis coefficients. The presence of large excess kurtosis coefficients, i.e. leptokurtosis, also indicates the probability of presence of outliers. Low level of $p$-value of Jarque-Bera test indicates severe non-normality conditional distribution of the series.

Decomposition of wavelets on the basis of discrete wavelet transform. Many research papers in the previous economic literature have analyzed the presence of short and long-run dynamics between oil price and exchange rate. According to Gallegati et al. (2011), a time series data comprises many periods which represent the appropriate time scales in an analysis. This necessitates the application of theory of signal which is widely used in the field of telecommunications or meteorology. 


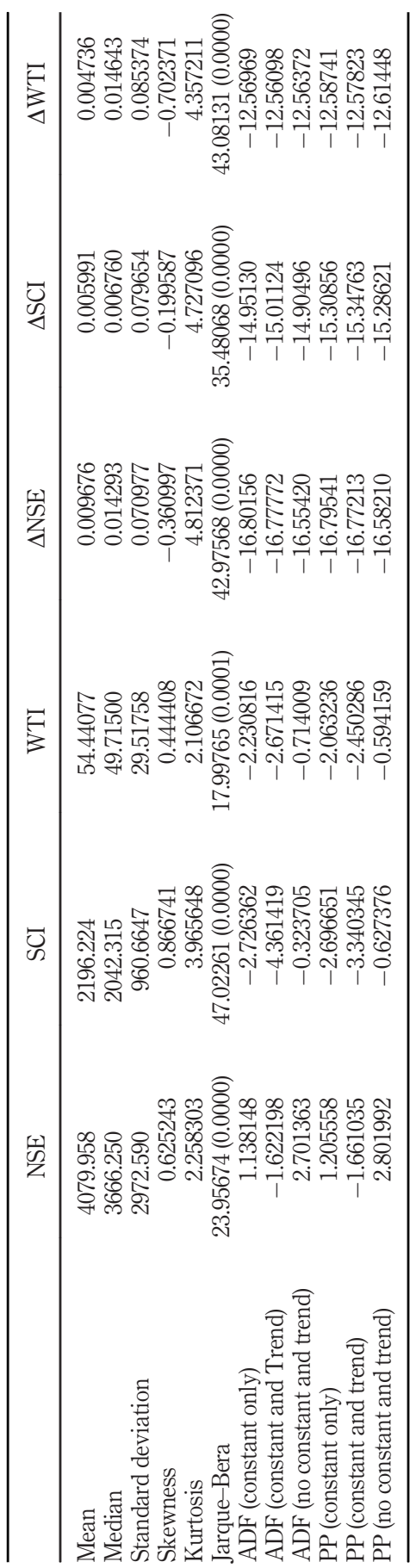

Crude oil price volatility

75

Table 1.

Descriptive statistics of variables at level and first-order difference 
XJM

19,1

76

To explore the relationship between WTI crude oil price and stock indices, the data series of the variables under study is decomposed based on DWT. The wavelet framework of analysis considers the non-stationary property of the variable as the inherent feature of the data which cannot be avoided by its preprocessing. The MRA of order $J=6$ of the data comprising of WTI crude oil price and stock indices i.e. NSE and SCI is undertaken by using MODWT based upon Daubechies' (1992) LA wavelet filter LA(8). The Daubechies' (1992) LA filter being able to provide most accurate time alignment between wavelet coefficients at original time series and various scales is the widely used wavelet.

The criteria for choosing the type and width of the wavelets are to strike a balance between two considerations. One consideration is that the width of wavelet filter should not be so short that it may lead to unrealistic blocks in the MRA. The other consideration is that it should not be so wide that it may lead to severe influence of boundary conditions, thus decreasing the localization of DWT coefficients and making the computation more difficult.

The MODWT plot for the variables is presented in Figures 1-3. The MODWT plot for WTI crude oil price shows the presence of great peaks in the original series during 2008 and 20122015, which is captured in D1-D4 components. For the MODWT plot of INRR (return series of Indian National Rupee) and CNYR (return series of Chinese Yuan), the great peaks can be observed in the original series during the year 1998 and 2014 and during the year 2008 and 2015, respectively, which is again captured in D1-D4 components for the decomposed variables. Similarly in the MODWT plot of NSE and SCI, the D1-D4 components of decomposed variable capture the peaks occurring during the year 2003 and year 2008 for NSE, and year 2003 and year 2014 for SCI.

Figure 1.

MODWT plot of decomposed series of WTI

\section{WTI}
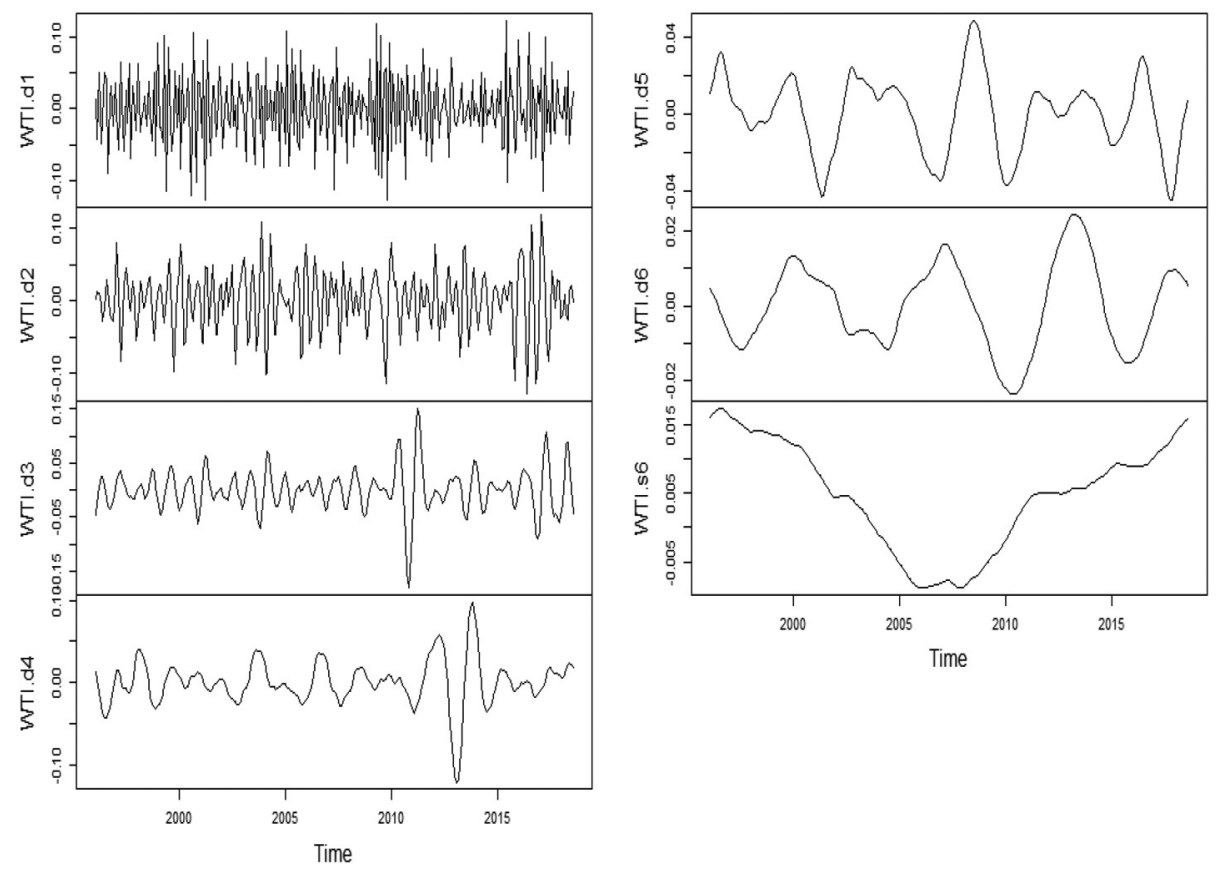
CNX
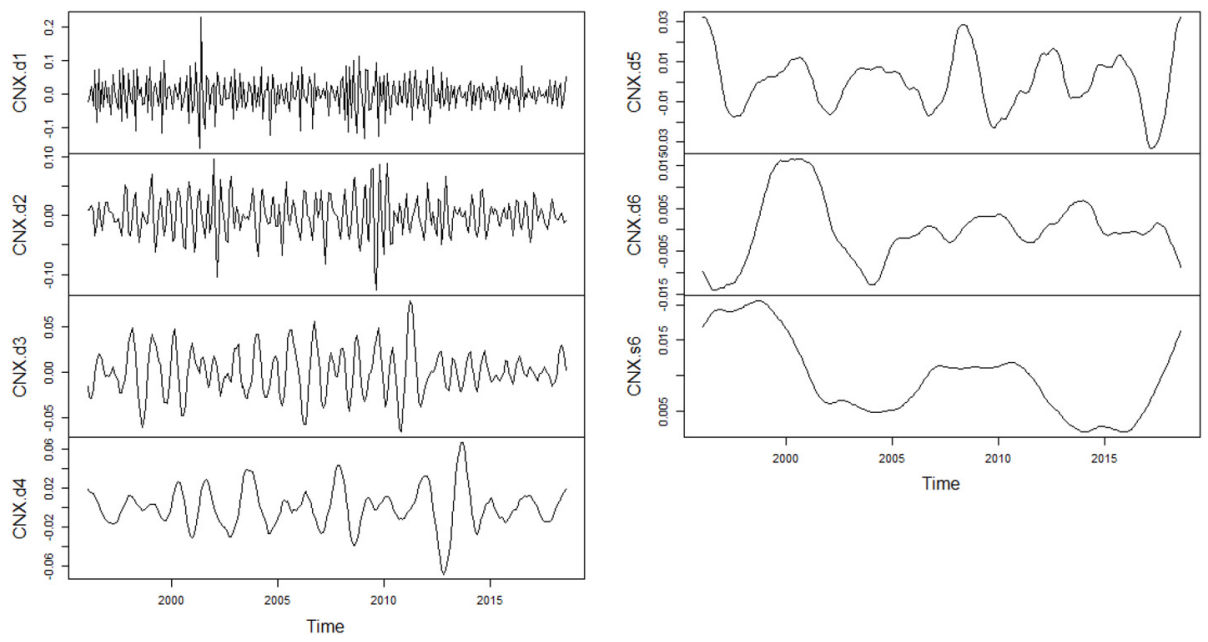

$\mathrm{SCl}$
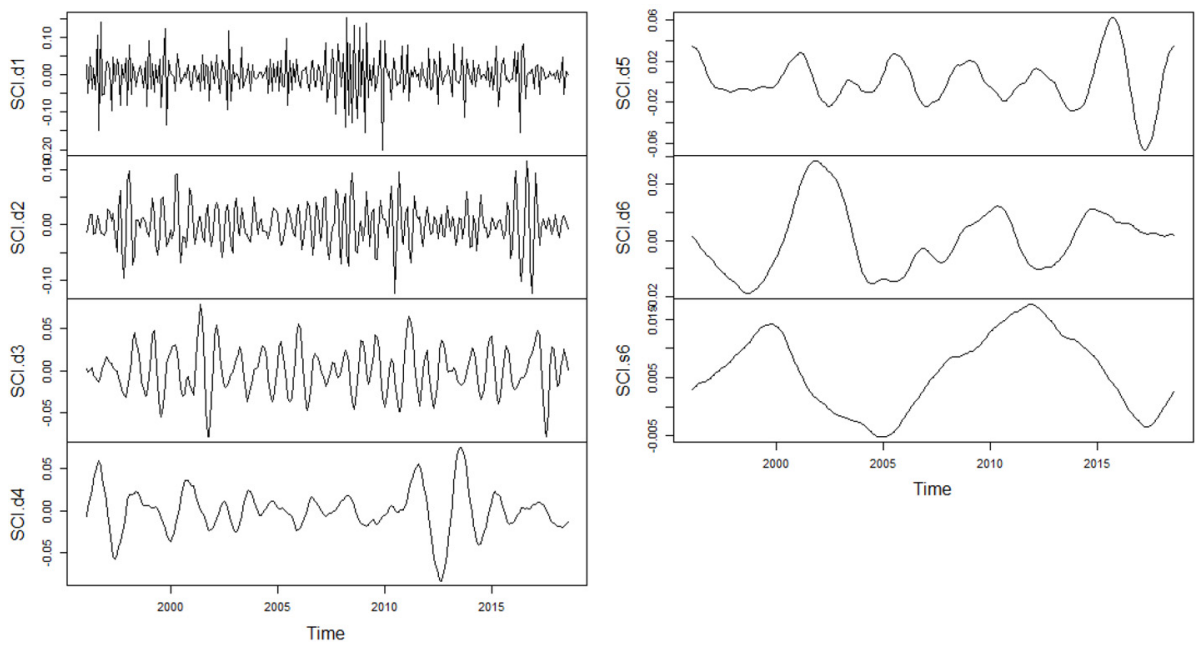

Crude oil price volatility

Figure 2.

MODWT plot of decomposed series of

NSE (CNX Nifty)

Figure 3.

MODWT plot of decomposed series of $\mathrm{SCI}$

After decomposing the variables, we examine the relative importance of short, medium and long-term dynamics of the variables under study. To examine the same, we use the energy of the variables' wavelet decomposition i.e. energy inherent in each scale. This energy is synonymous to variance and is represented as percentage of overall energy. Thus, this percentage of whole energy is explaining the percentage of variance being explained by each scale. According to Percival and Walden (2000), DWT can decompose energy in a time series across scales. However, MODWT is termed as energy preserving platform i.e. time series variance is preserved in variance of coefficients obtained from MODWT (Percival and Mofjeld, 1997). 
$\mathrm{XJM}$

19,1

78

The energy of each scale expressed as percentage of overall energy for the variables under study are presented in Table 2. For obtaining unbiased estimator, we do not take into account the coefficients affected by the boundaries. Here we use six scales, with the seventh scale being included in the smooth. For the energy decomposition and energy distribution, we use Daubechies' (1992) LA filters as they are least affected by the boundaries.

In Table 2, the wavelet scales are presented in first column. The energy distribution of WTI crude oil price and stock indices, CNX Nifty and SCI are presented in subsequent columns of Table 2 . The discussion of energy distribution can be categorized into four major periods, namely, short run (D1 + D2), medium run (D3 + D4), long run (D5 + D6) and very long run (S6). From the energy decomposition table, we can witness short run dominating all other periods and frequencies which explain most of the variances for oil price $(80 \%)$, CNX Nifty $(86 \%)$ and SCI (84\%). The outcome from the energy decomposition reflects the importance of high frequency variations and seasonality components of the variables under study.

To examine the association between WTI crude oil price and stock indices, CNX Nifty and SCI, we use wavelet covariance analysis to separate the effect across time scale and frequency bands. The MODWT-based wavelet covariance between WTI crude oil price and CNX Nifty and SCI is illustrated in Figure 4.

From Figure 4, we can observe wavelet covariance between WTIR (return series of West Texas Instrument crude oil price) and dependent variables under study slowly fluctuating in the analyzed period, showcasing the flattening tendency in the long-run interval. In the wavelet covariance between WTI global crude oil price and stock indices of CNX Nifty (CNXR) for India and SCI (SCIR) for China, we observe positive covariance between the dependent and independent variable across all the levels of wavelet decomposition. This

Table 2.

Energy

decomposition for WTI crude oil price and stock indices CNX Nifty and SCI

\begin{tabular}{lrrr}
\hline Wavelet scales & WTIP & CNXP & SCIP \\
\hline D1 (2-4) & 34.239 & 50.935 & 44.224 \\
D2 (4-8) & 21.016 & 21.566 & 22.734 \\
D3 (8-16) & 25.321 & 11.978 & 11.510 \\
D4 (16-32) & 10.879 & 8.341 & 10.245 \\
D5 (32-64) & 5.548 & 3.654 & 7.916 \\
D6 (64-128) & 1.822 & 1.088 & 2.123 \\
S6 (above 128) & 1.176 & 2.438 & 1.247
\end{tabular}

Notes: WTIP = positive changes in WTI Crude Oil Price; $\mathrm{CNXP}=$ positive changes in CNX Nifty Index; $\mathrm{SCIP}=$ positive changes in Shanghai Composite Index

Figure 4.

Wavelet covariance between WTIR and CNXR and SCIR

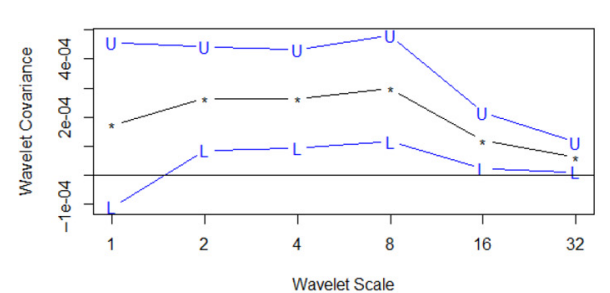

(a)

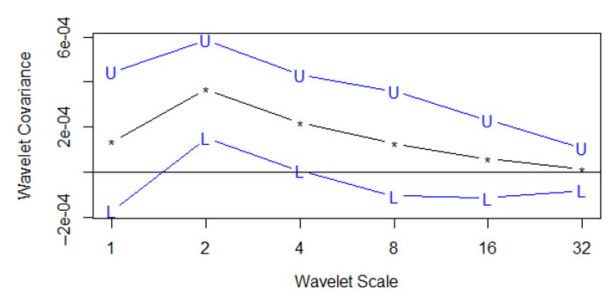

(b)
Notes: (a) Wavelet Covariance between WTIR and CNXR; (b) Wavelet Covariance between WTIR and SCIR 
indicates increase in stock price or development of stock indices associated with increase in oil price. The positive association between WTI global crude oil price and stock indices of India and China is strong at short and medium run.

The wavelet correlation between the WTI global crude oil price stock indices of India and China across different scales is illustrated in Figure 8. In the short and medium level of decomposition, we observe less fluctuation in the wavelet correlation among the variables, which increases in the long run. However, in case of wavelet correlation between WTI crude oil price and SCI, there is less fluctuation across all the levels of decomposition. The wavelet correlation between WTI crude oil volatility price and CNX Nifty and SCI is positive across all the levels of decomposition (Figure 5).

The given conventional approach of wavelet covariance and correlation analysis for deriving the flow of information or direction of causality between the variables could be misleading. The cross-correlation being a symmetric approach may not be considered suitable approach to identify the lead-lag relationship between the variables in the systems comprising of feedback. This necessitates the adoption of Granger causality test for identifying the flow of information between the variables. Moreover apart from DWT approach, continuous wavelet transform (CWT) approach is also used.

\section{Continuous wavelet transform approach}

In DWT, we although combine the time and frequency analysis, but we face an arduous task in interpreting it. The difficulty in interpretation of time and frequency analysis in DWT can be attributed to difference in the resolution of the frequency information at every stage. To remove this bottleneck, we can use continuous analysis which helps in reinforcing the traits and making the information more visible. We perform the CWT to check the robustness of the results obtained from DWT and interpret its findings. While analyzing the relationship between WTIR and stock indices of India and China, we interpret the findings through power spectrum (Figure 6) of all the series under study, and cross wavelet transform (XWT) and wavelet coherence (WTC) between the series under study (Figures 7 and 8).

The wavelet power spectrum of WTI crude oil price and stock indices is represented in contour plot along three dimensions i.e. time, frequency (period) and color code (Figure 6). In WTI global crude oil price, the strong variance can be observed in short run during the period from March 2000 to May 2003. Similarly, the strong variance becomes prominent in in medium and long run during the time period between July 2008 and June 2009 and in medium run during the time period from September 2014 to March 2015. In stock index CNX Nifty, the variance is found in the short run during March 2000-2001 and during the time period between July 2008 and 2009. In the medium run, the same is observed during May 2004. In SCI, very little variance is observed in the short run. However, in the medium and long run, the variance becomes significant during the time period between May 2004 and August 2009.

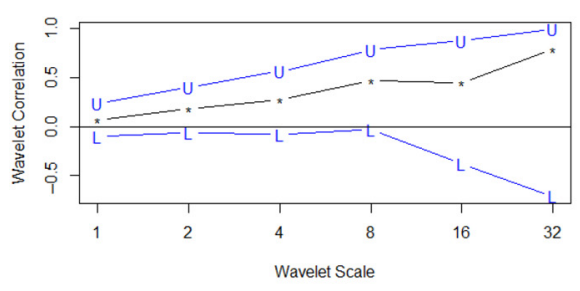

(a)

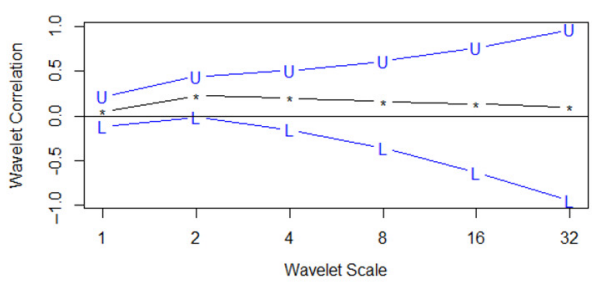

(b)

Notes: (a) Wavelet Covariance between WTIR and CNXR; (b) Wavelet Covariance between WTIR and SCIR 
XJM

19,1

80

Figure 6.

Wavelet power spectrum of variables under study
Figure 7.

XWT between WTI crude oil prices and stock indices of India and China
Figure 8.

WTC between WTI crude oil prices and exchange rate and stock indices of India and China
We further analyze the XWT between the variables under study. The XWT reveals the common features and the relative phase between the series in time-frequency phase. From the XWT between WTI and stock indices of India and China, we observe very few arrows oriented toward the left direction i.e. anti-phase direction. From the XWT, we can figure out which variable under study is leading or lagging.

The major drawback which XWT suffers from is that it can describe the two series' common power without normalizing the single-wavelet power spectrum. Thus, even if one
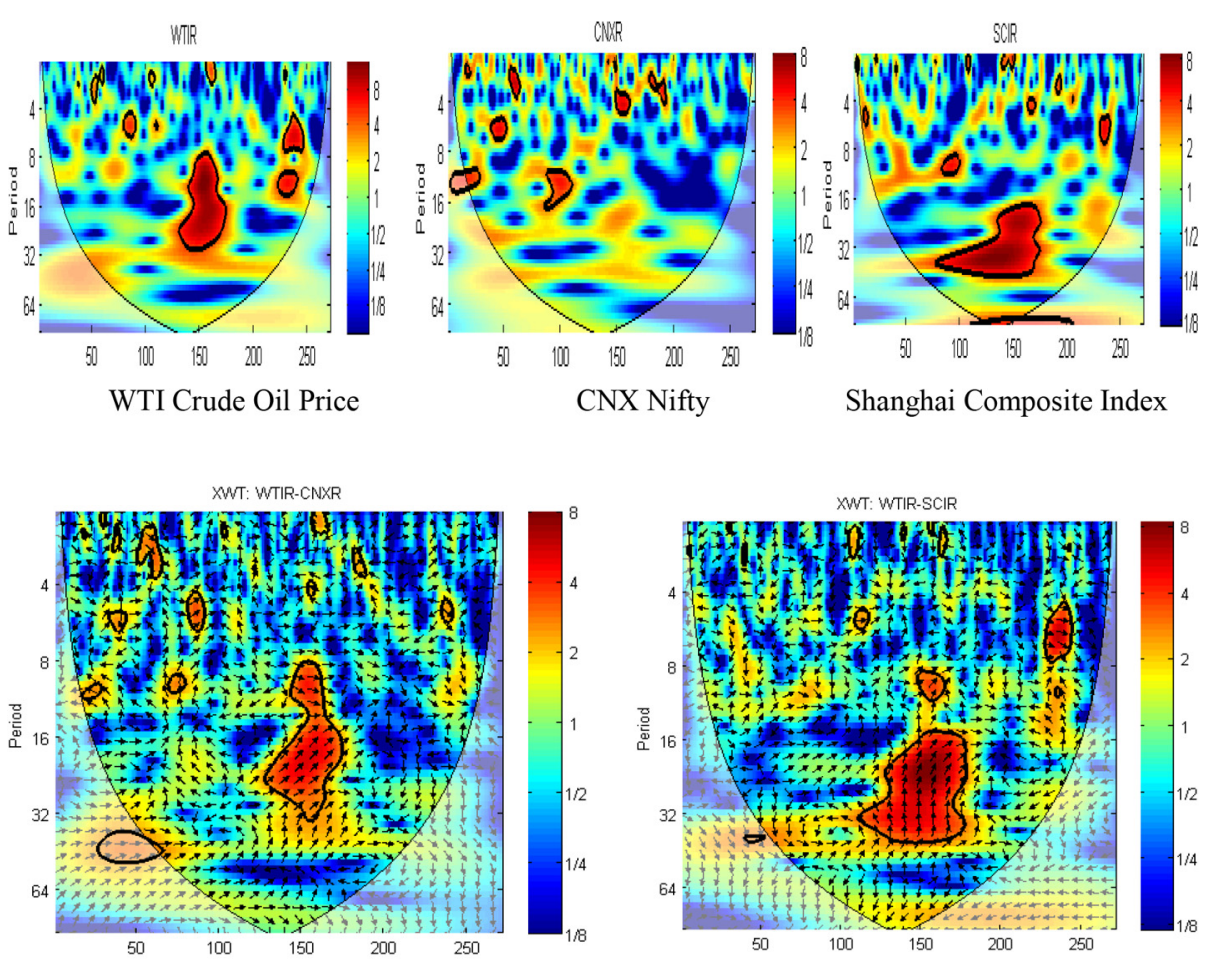

XWT: WTIR-CNXR

XWT: WTIR-SCIR

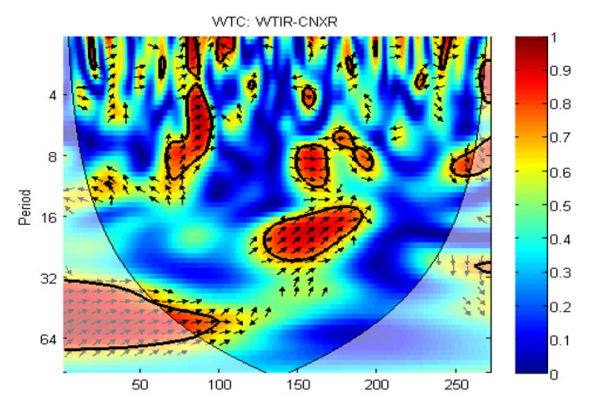

WTC: WTIR-CNXR

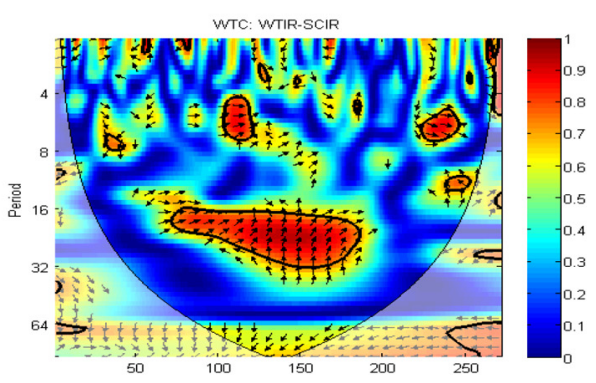

WTC: WTIR-SCIR 
spectrum is local, the second spectrum produces strong peaks which can be formed despite the possible absence of any relationship between the series.

After the XWT analysis, we further move on to perform WTC analysis between the crude oil price and stock indices (CNXR and SCIR) of India and China. The WTC analysis between the variables is illustrated in Figure 8.

While analyzing the wavelet transform coherence (WTC) between WTI crude oil price and stock index CNX Nifty, in the short and medium-run causality runs from CNX Nifty (CNXR) to WTIR during the time period 2001-2002, and similarly in the medium run during the time period around 2004-2005, we observe in phase causality as well as causality running from CNXR to WTIR. During certain periods, the causality also runs from WTIR to CNXR. In the short run, during the time period 2013-2014, although the causality runs from WTIR to CNXR, the degree of causality is insignificant. When we analyze the WTC between WTIR and SCI, we observe causality between the variables running from both the directions. In the short run during the time period around 2004 and 2012-2013, the causality between the variables appear to be in-phase, i.e. running in same direction. In the medium run during the time period 20052012, the causality from SCIR to WTIR is significant. However, in the short and medium run during the time period 2005-2006, the causality running from WTIR to SCIR appears but is not much significant. The findings from the WTC analysis are further substantiated by waveletbased Granger causality analysis between the variables.

\section{Wavelet-based Granger causality analysis}

We further analyze the relationship between WTIR and stock indices of India and China with Granger causality analysis at different time scales. The findings from the waveletbased Granger causality analysis are presented in Tables 3 and 4.

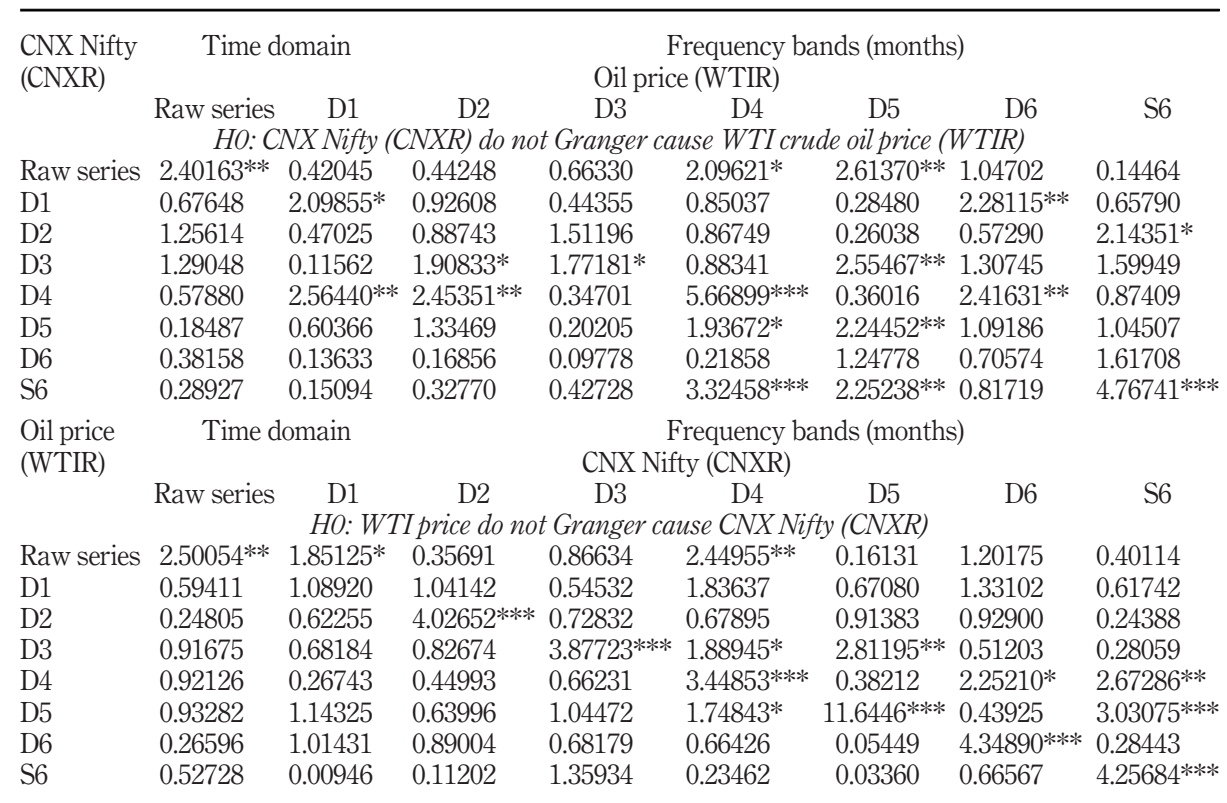

Crude oil price volatility

Notes: *,**,*** represents significance at $10 \%, 5 \%$ and $1 \%$ significance level respectively

Table 3.

Wavelet-based Granger causality test between WTIR and CNXR 


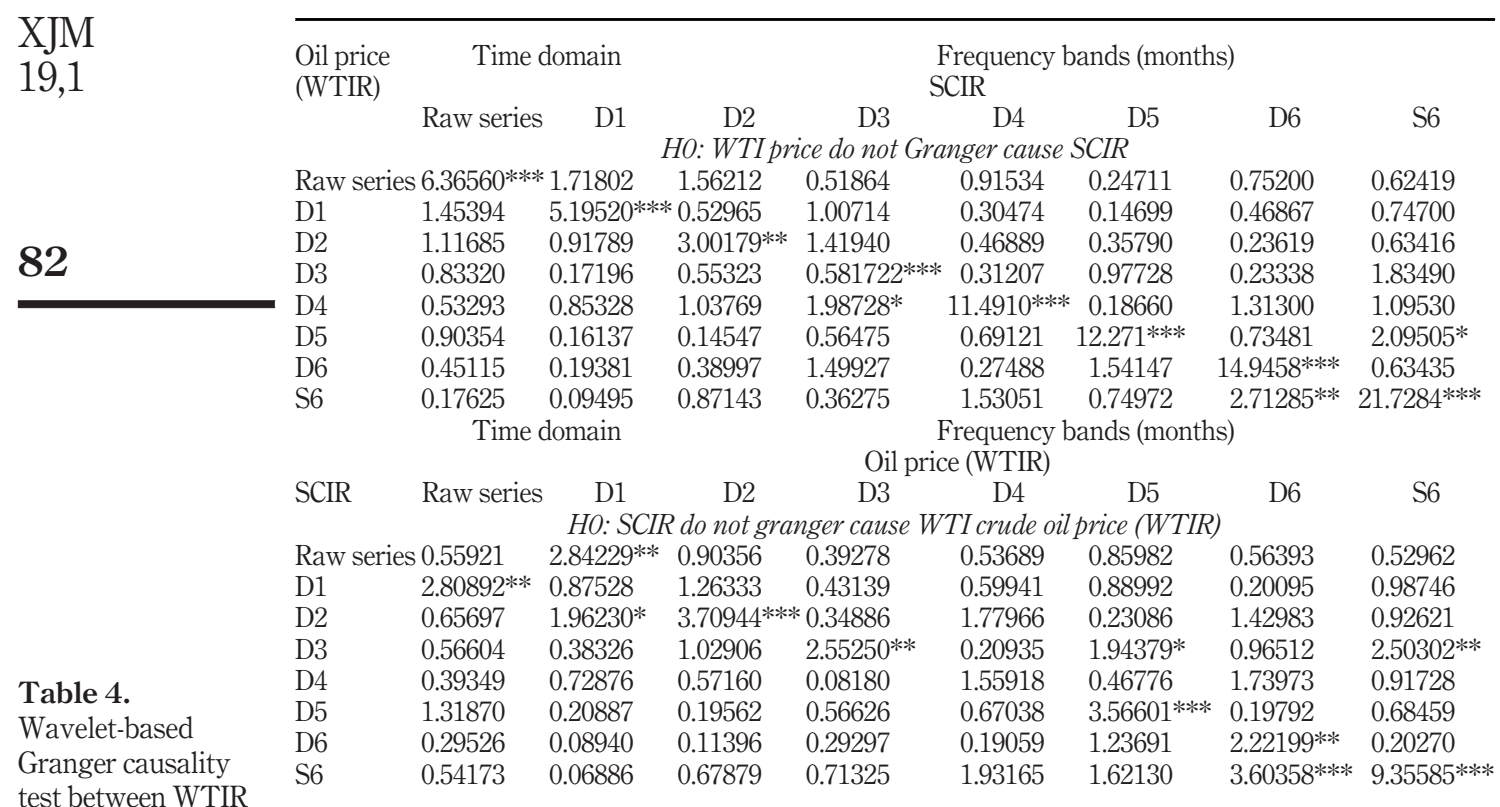
and SCIR

Notes: $*, * *, * * *$ represents significance at $10 \%, 5 \%$ and $1 \%$ significance level respectively

The results of the wavelet-based Granger causality test between WTI crude oil price and stock indices of India and China reinforces the findings obtained from WTC between the variables. There is a marked variability in the causality between the variables across the different frequency ranges and time scales.

We test the causality among the variables at different cross-frequencies, to analyze whether high, medium or low frequencies of oil price Granger cause high, medium or low frequencies of stock indices CNX Nifty and SCI. From the wavelet-based Granger causality analysis, we observe in almost all the cases, a given frequency band in WTI global crude oil price defines the similar cycle in stock indices CNX Nifty and SCI. This in turn leads to one variable's structural changes causing short run fluctuations in another variable and vice versa.

The wavelet-based Granger causality analysis between WTI crude oil price and CNX Nifty is illustrated in Table 3. There exists a bidirectional causality between raw series of WTI crude oil price and CNX Nifty at $5 \%$ significance level. When we decompose the time series data of both the variables, the pattern of causality between the variables start varying at different levels. There exists a bidirectional causality between the raw series of WTI crude oil price and CNX Nifty at 5\% significance level. At D1 decomposition level, WTI crude oil price do not have statistically significant influence on CNX Nifty. However, CNX Nifty at D1 decomposition level influence crude oil price in the long run at 5\% significance level. At D2 decomposition level, the changes in WTI crude oil price influence CNX Nifty in the long run, but CNX Nifty does not have any statistically significant influence on the oil price. At D3 decomposition level, WTI crude oil price influence CNX nifty at both medium and long run; however, the reverse is not statistically significant. At D4 decomposition level of both the series, there exists a bidirectional causality between the variables at medium and 
long run. Again at D5 and D6 decomposition level, the WTI crude oil price influences CNXR at long and very long run, but CNX Nifty does not have any statistically significant influence on the crude oil price. At the $\mathrm{S} 6$ decomposition level, there exists a bidirectional causality between the variables at both long and very long run.

In case of China (Table 4), when we analyze the raw series data of both the variables, we find unidirectional causality from WTI crude oil price to SCIR. On decomposing the time series of both variables, we find at D1 decomposition level, that WTI crude oil price Granger cause SCIR in the short run, but SCIR do not have any statistically volatility significant influence on the oil price. At D2 decomposition level, although the influence of WTI crude oil price on SCIR is not statistically significant, but is influenced by D2 decomposition level of SCIR in the short run. At D3, D4, D5, D6 and S6 decomposition level, we use statistically significant Granger causality from WTI crude oil price to SCIR at their respective frequency bands. At D5 and S6 decomposition level of SCIR, there exists a bidirectional causality between SCIR and WTI crude oil price in the long run and very long run.

We can sum up with the fact that with the wavelet decomposition, we can identify the time frequencies, in which the causality relationship between the WTI crude oil price and stock indices of India and China manifests itself. The wavelet decomposition helps us to analyze the influence of WTI crude oil price on the stock indices both in short run and long run. The wavelet-based Granger causality analysis revealed the causality between the variables existing both in the short run and long run.

\section{Concluding observations}

The present study examines the causality between changes in global crude oil price and stock indices of India and China under wavelet transform framework. Under the given framework, the time series of the WTI global crude oil price and stock indices of India and China i.e. CNX Nifty and SCI is decomposed for the above said analysis.

In the given aspect, we initially used wavelet covariance and correlation to explore the influence of WTI global crude oil price on the real effective exchange rate and stock indices of India and China. The wavelet covariance between WTIR and stock indices of India and China, i.e. CNXR and SCIR, is positive across all the levels of decomposition. The wavelet covariance between WTIR and SCIR is strong at short and medium run. The wavelet correlation between WTIR and stock indices of India and China, i.e. CNXR and SCIR, is positive across all the levels of decomposition.

The CWT approach helps in exploring the variance in the crude oil price and stock indices of India and China. In the analysis, we observe strong variance in WTIR in short, medium and long run at various time periods. The variance in CNXR, the stock index for India, is observed in short and medium run at various time periods. The Chinese stock index, i.e. SCIR, experiences very little variance in short run and significant variance in long and medium run during May 2004 and August 2009. The WTC analysis indicates that causality from WTIR to CNXR though exists but is insignificant. Similarly, we find causality between Chinese stock index SCIR and WTIR running from both the directions. Here, also the causality from WTIR to SCIR does not appear to be much significant.

The wavelet-based Granger causality analysis further substantiates the finding from WTC analysis. The raw time series of WTIR Granger cause raw time series of CNX at $5 \%$ level of statistical significance. The decomposed time series of both the variables granger cause each other at medium long and very long run. The similar scenario is also observed 
between WTIR and Chinese stock index SCIR where again the bidirectional causality between the variables exist at medium, long and very long run.

\section{References}

Anoruo, E. (2010), "Testing for linear and nonlinear causality between crude oil price changes and stock market returns", International Journal of Economic Sciences and Applied Research, Vol. 4 No. 3, pp. 75-92.

Anoruo, E. and Mustafa, M. (2007), "An empirical investigation into the relation of oil to stock market prices", North American Journal of Finance and Banking Research, Vol. 1 No. 1, pp. 22-36.

Basher, S. and Sadorsky, P. (2006), "Oil price risk and emerging stock markets", Global Finance Journal, Vol. 17 No. 2, pp. 224-251.

Basher, S.A., Haug, A.A. and Sadorsky, P. (2012), "Oil prices, exchange rates and emerging stock markets”, Energy Economics, Vol. 34 No. 1, pp. 227-240.

Benassy-Quere, A., Mignon, V. and Penot, A. (2007), "China and the relationship between the oil price and the dollar", Energy Policy, Vol. 35 No. 11, pp. 5795-5805.

Bhar, R. and Nikolova, B. (2009), "Oil prices and equity returns in the BRIC countries", World Economy, Vol. 32 No. 7, pp. 1036-1054.

Boyer, M. and Filion, D. (2004), "Common and fundamental factors in stock returns of Canadian oil and gas companies”, Energy Economics, Vol. 29 No. 3, pp. 428-453.

Chen, N., Roll, R. and Ross, S.A. (1986), "Economic forces and the stock market", The Journal of Business, Vol. 59 No. 3, pp. 383-403.

Chittedi, K.R. (2012), “Do oil prices matters for Indian stock markets? An empirical analysis”, Journal of Applied Economics and Business Research, Vol. 2 No. 1, pp. 2-10.

Cong, R.G., Wei, Y.M., Jiao, J.L. and Fan, Y. (2008), "Relationships between oil price shocks and stock market: an empirical analysis from China", Energy Policy, Vol. 36 No. 9, pp. 3544-3553.

Cornish, C.R., Bretherton, C.S. and Percival, D.B. (2006), "Maximal overlap wavelet statistical analysis with application to atmospheric turbulence", Boundary-Layer Meteorology, Vol. 119 No. 2, pp. 339-374.

Cunando, J. and De Gracia, F.P. (2014), "Oil price shocks and stock market returns: evidence for some European countries”, Energy Economics, Vol. 42, pp. 365-377.

Daubechies, I. (1992), Ten Lectures on Wavelets, SIAM, Philadelphia.

El-Sharif, I., Brown, D., Burton, B., Nixon, B. and Russell, A. (2005), "Evidence on the nature and extent of the relationship between oil prices and equity values in the UK", Energy Economics, Vol. 27 No. 6, pp. 819-830.

Faff, R. and Brailsford, T.J. (1999), “Oil price risk and the Australian stock market”, Journal of Energy Finance \& Development, Vol. 4 No. 1, pp. 69-87.

Fayoumi, N.A. (2009), "Oil prices and stock market returns in oil importing countries: the case of Turkey, Tunisia and Jordan", European Journal of Economics, Finance and Administrative Sciences, Vol. 16 No. 2, pp. 86-101.

Filis, G. (2010), "Macro economy, stock market and oil prices: do meaningful relationships exist among their cyclical fluctuations?", Energy Economics, Vol. 32 No. 4, pp. 877-886.

Gay, R. Jr., (2008), "Effect of macroeconomic variables on stock market returns for four emerging economies: Brazil, Russia, India, and China”, International Business \& Economics Research Journal, Vol. 7 No. 3, pp. 1-8.

Gallegati, M., Gallegati, M., Ramsey, J. and Sr, W. (2011), "The US wage Phillips curve across frequencies and over time", Oxford Bulletin of Economics and Statistics, Vol. 73, pp. 489-508.

Gencay, R., Selcuk, F. and Whitcher, B. (2002), An Introduction to Wavelets and Other Filtering Methods in Finance and Economics, Academic Press, San Diego, CA. 
Gogineni, S. (2007), "The stock market reaction to oil price changes", Working Paper, University of Oklahoma, Norman, OK.

Hammoudeh, S. and Li, H. (2005), "Oil sensitivity and systematic risk in oil-sensitive stock indices",

Crude oil price volatility Journal of Economics and Business, Vol. 57 No. 1, pp. 1-21.

Huang, R.D., Masulis, R.W. and Stoll, H.R. (1996), "Energy shocks and financial markets”, Journal of Futures Markets, Vol. 16 No. 1, pp. 1-27.

Jones, C.M. and Kaul, G. (1996), "Oil and the stock markets", The Journal of Finance, Vol. 51 No. 2, pp. 463-491.

Jones, D.W., Leiby, P.N. and Paik, I.K. (2004), "Oil price shocks and the macroeconomy: what has been learned since 1996?”, The Energy Journal, Vol. 25 No. 2, pp. 1-32.

Lescaroux, F. and Mignon, V. (2008), "On the influence of oil prices on economic activity and other macroeconomic and financial variables", OPEC Energy Review, Vol. 32 No. 4, pp. 343-380.

Lin, C.C., Fang, C.R. and Cheng, H.P. (2010), "Relationships between oil price shocks and stock market: an empirical analysis from greater China”, China Economic Journal, Vol. 3 No. 3, pp. 241-254.

Maghyereh, A. (2004), "Oil price shocks and emerging stock markets: a generalized VAR approach", International Journal of Applied Econometrics and Quantitative Studies, Vol. 1 No. 2, pp. 27-40.

Masih, R., Peters, S. and De Mello, L. (2011), "Oil price volatility and stock price fluctuations in an emerging market: evidence from South Korea", Energy Economics, Vol. 33 No. 5, pp. 975-986.

Mensi, W., Hammoudeh, S. and Kang, S.H. (2017), "Risk spillovers and portfolio management between developed and BRICS stock markets", The North American Journal of Economics and Finance, Vol. 41, pp. 133-155.

Mohanty, S.K., Nandha, M., Turkistani, A.Q. and Alaitani, M.Y. (2011), "Oil price movements and stock market returns: evidence from Gulf Cooperation Council (GCC) countries”, Global Finance Journal, Vol. 22 No. 1, pp. 42-55.

Nandha, M. and Faff, R. (2008), "Does oil move equity prices? A global view", Energy Economics, Vol. 30 No. 3, pp. 986-997.

Narayan, K.P. and Narayan, S. (2010), "Modelling the impact of oil prices on Vietnam's stock prices", Applied Energy, Vol. 87 No. 1, pp. 356-361.

O'Neil, T.J., Penm, J. and Terrell, R.D. (2008), "The role of higher oil prices: a case of major developed countries", Research in Finance, Vol. 24, pp. 287-299.

Pappetrou, E. (2001), "Oil price shocks, stock market, economic activity and employment in Greece", Energy Economics, Vol. 23, pp. 511-532.

Percival, D.B. (1995), "On estimation of the wavelet variance”, Biometrika, Vol. 82 No. 3, pp. 619-631.

Percival, D.B. and Mofjeld, H.O. (1997), "Analysis of subtidal coastal sea level fluctuations using wavelets”, Journal of American Statistical Association, Vol. 92 No. 439, pp. 868-880.

Percival, D.B. and Walden, A.T. (2000), Wavelet Methods for Time Series Analysis, Cambridge University Press, Cambridge, UK.

Ramsey, J.B. (1999), "The contribution of wavelets to the analysis of economic and financial data", Philosophical Transactions of the Royal Society of London, Vol. 357 No. 1760, pp. 2593-2606.

Sadorsky, P. (1999), "Oil price shocks and stock market activity", Energy Economics, Vol. 21 No. 5, pp. 449-469.

Sadorsky, P. (2001), "Risk factors in stock returns of Canadian oil and gas companies", Energy Economics, Vol. 23 No. 1, pp. 17-28. 
XJM

19,1

Sahu, T.N., Mondal, D. and Bandopadhyay, K. (2014), "An empirical study on the dynamic relationship between the oil price and Indian stock market”, Managerial Finance, Vol. 40 No. 2, pp. 200-215.

Sari, R. and Soytas, U. (2006), "The relationship between stock returns, crude oil prices, interest rates, and output: evidence from a developing economy", The Empirical Economics Letters, Vol. 5 No. 4, pp. 205-220.

Sehgal, S. and Kapur, R. (2012), "Relationship between oil price shocks and stock market performance: evidence for select global equity markets", Vision: The Journal of Business Perspective, Vol. 16 No. 2, pp. 81-92.

Sharma, N. and Khanna, K. (2012), "Crude oil price velocity and stock market ripple - a comparative study of BSE with NYSE \& LSE”, Indian Journal of Exclusive Management Research, Vol. 2 No. 7, pp. 1-7.

Toraman, C., Basarir, C. and Bayramoglu, M.F. (2011), "Effects of crude oil price changes on sector indices of Istanbul stock exchange", European Journal of Economic and Political Studies, Vol. 4 No. 2, pp. 109-124.

Valadkhani, A., Chancharat, S. and Havie, C. (2009), "Analysing the impact of international stock markets and macroeconomic variables on the Thai stock market", The Business Review, Vol. 12 No. 2, pp. 50-56.

Wei, C. (2003), "Energy, the stock market, and the putty-clay investment model", American Economic Review, Vol. 93 No. 1, pp. 311-323.

Yurtsever, C. and Zahor, T. (2007), "Oil price shocks and stock market in The Netherlands", Working paper, University of Groningen, Groningen.

\section{Further reading}

Miller, J.I. and Ratti, R.A. (2009), "Crude oil and stock markets: stability, instability, and bubbles", Working Paper, University of Missouri, Columbia.

\section{Corresponding author}

Shekhar Mishra can be contacted at: shekhar.ximb2019@gmail.com

For instructions on how to order reprints of this article, please visit our website: 